\title{
Design solutions for structures with optimal internal stress distribution
}

\author{
Yurii Kitov ${ }^{1}$, Maryna Verevicheva ${ }^{1}$, Glib Vatulia ${ }^{1 *}$, Yevhen Orel $^{1}$, and Svitlana Deryzemlia ${ }^{1}$ \\ ${ }^{1}$ Ukrainian State University of Railway Transport, Structural Mechanics and Hydraulics Department, 7 Feuerbach Sq., \\ 61050 Kharkiv, Ukraine
}

\begin{abstract}
The development of transport infrastructure, reconstruction of roads and railways, together with growing urban traffic necessitate the construction of new bridges and/or the reconstruction of existing ones. The primary objective of design is obtaining a reasonable system in compliance with the requirements for stress-strain state: equal strength or equal torque, maximum rigidity or minimum weight of the structure. In the paper, the object of optimization is the design of a three-span girder bridge of a specified length under the constant load. The sets of both statically determinate and statically indeterminate systems are considered, namely: three-span hinged girders in which the hinge joints are located in the end spans or middle span, and three-span continuous girders on unilateral supports or on resilient supports. Two variable design parameters are taken into account: the length of each end span $z_{1}$ and the position of the hinges $x$ for the statically determinate systems, and the displacement of support assemblies joints $\Delta$ or the value that affects the displacement, i.e. flexibility of yielding supports $c$ for the statically indeterminate ones. The authors proved that there are optimal solutions in the set of statically indeterminate systems both with unilateral and with elastic constraints.
\end{abstract}

\section{Introduction}

Along with the development of new constructive solutions and methods for assessment of the stress-strain state and bearing capacity of building structures, the issue of optimizing their parameters is also addressed now.

In [1] three principles are described that underlie the design of steel constructions: metal saving, reduction of labour intensity of manufacture and installation time. In different periods, these principles were taken into account in different ways, depending on the state-of-theart in the industry and the urgent needs of society, as well as the existing structural engineering methods. Before methods of optimization appeared, methods of determining the weight depending on the design parameters of structures were developed. Afterwards, methods of determining the labour intensity of manufacture and the installation of structures were also developed.

The beginning of the modern epoch of optimization of building constructions dates back to more than 70 years ago when the expediency of application of developed mathematical programming methods in this area was substantiated [1-7].

Along with the obtained applied results, these works contain the analysis of the principal quality criteria, restriction types, as well as problem-solving techniques for optimal design.
Initially, the only tasks in using the mathematical programming methods with problems of steel structure optimization were to minimize the girder and the plane frame weight. Further, problem statements got more complicated, in particular, including the transition to the economic optimality criteria, adjustment of the stressstrain state of construction, unification and typification of elements, which finally allowed bringing the optimization theory closer to the design practice in engineering, transport and construction [8-17].

\section{General provisions}

We assume that the optimal internal force distribution in a structure is the set of internal forces in which the system becomes optimal, for example, a system of minimum volume or cost.

One of the optimal design types is systems that have strength conditions as limitations. The construction is optimal, if the maximum stresses in all its elements are equal to the estimated ones, that is, a system has equal strengths. If the stress-strain state of all elements is the same, and their cross-sections are equal, then the system can be considered to have equal strength if the forces in the nominal cross-sections are equal. To obtain the optimal system with equal strengths, equal forces of the elements should be obtained by changing the geometric characteristics of those elements.

\footnotetext{
* Corresponding author: glebvatulya@gmail.com
} 
All constructions can be conditionally divided into two sets: the set of statically determinate systems and the set of statically indeterminate ones.

In the set of statically determinate systems, the forces are determined by means of the static equilibrium equations; their distribution at the given stress depends only on the linear dimensions of elements and their mutual arrangement, but not on the displacement of the support constraints.

In the set of statically indeterminate systems, those forces are also determined by means of equilibrium equations, as well as by equations of strain compatibility. Their distribution depends both on the linear dimensions of the elements and on the displacement of the support constraints. Therefore, in the set of statically indeterminate systems, an optimal system can be obtained not only by changing the linear dimensions of elements, but also by displacing the support constraints. For this purpose, the system - for example, a continuous girder - must have unilateral support constraints or resilient supports.

To determine forces in the set of statically indeterminate systems, additional equations are required along with equilibrium equations, namely, strain compatibility equations. Hence, the force distribution depends both on the linear dimensions of the elements and on their deformations. Therefore, equal strength of all elements cannot be achieved only by changing their linear dimensions.

Let us use an example to show that in the set of statistically indeterminate systems with unilateral as well as elastic constraints, optimal solutions exist also under constant load. They completely coincide with the solutions in the set of statically determinate systems.

\section{Optimization of the design of the three-span girder bridge}

\subsection{Problem statement}

An optimal design of a three-span girder bridge of a given length $l$ under constant load $q=$ const should be obtained. The cross-section of the girder is constant and equal in all spans, the layout is symmetrical.

Let us consider four design options: two of them belong to the statically determinate systems and the other two - to the statically indeterminate ones (Fig. 1).

The first two are three-span statically determinate hinge-connected girders in which hinge joints are located in the end spans (Fig. 1, a) or in the middle span (Fig. 1, b).

The second two are three-span continuous girders on unilateral supports (Fig.1, c) or on resilient supports (Fig. 1, d).

There are two design parameters. The first one which effects the force distribution is the length of each end span $z_{1}$, which is a common parameter for all types of girders. The second parameter is specific for each set: in the statically determinate systems, it determines the position of hinges $x$; in the statically indeterminate systems, it is displacement of the support joints $\Delta$ or the value effecting the displacement, i.e. flexibility of resilient supports $c$.

\subsection{Optimization conditions}

Since the load bearing elements are girders of constant cross-section, they are in the equal strain-strain states under load. Therefore, the optimal bridge design is equally strong girders with equal breakdown torques in the characteristic cross-sections: in the first girder, above the support and in the second girder (Fig. 1).

There are three characteristic cross-sections with breakdown torques; therefore, two optimality conditions are required, namely:

$$
\begin{aligned}
& M_{1 \max }=\left|M_{\text {opt }}\right|, \\
& \left|M_{\text {opt }}\right|=M_{2 \max } .
\end{aligned}
$$

On the basis of the above conditions, optimality equations are derived; there are two of them for this problem. We can determine the optimal design parameters for different types of girders by solving these equations together.

\subsection{Continuous girder with hinge joints in the end spans}

For such a structure (Fig. 1, a), let us write:

$$
\begin{gathered}
M_{1 \max }=\frac{q x^{2}}{8}, M_{o p t}=\frac{q z_{1}\left(x-z_{1}\right)}{2}, \\
M_{2 \max }=q\left[\frac{x z_{1}}{2}+\frac{l\left(l-4 z_{1}\right)}{8}\right] .
\end{gathered}
$$

From (1), we can obtain the expression for $x$ :

$$
x=2 z_{1}(\sqrt{2}-1) .
$$

Parameter $z_{1}$ can be numerically determined by exhaustive search with 0,001 increment until the condition (2) is met.

\subsection{Continuous girder with hinge joints in the middle span}

We can write the equations for such a structure (Fig.1,b):

$$
\begin{gathered}
M_{1 \max }=\frac{q}{8 z_{1}^{2}}\left[\left(x+z_{1}\right)^{2}-l x\right], \\
M_{\text {opt }}=-\frac{q x\left(l-2 z_{1}-x\right)}{2}, M_{2 \max }=q \frac{\left[l-2\left(x+z_{1}\right)\right]^{2}}{8} .
\end{gathered}
$$

From (2), we can obtain the expression for $x$ :

$$
x=\frac{\sqrt{2}-1}{2 \sqrt{2}}\left(l-2 z_{1}\right) .
$$


Parameter $z_{1}$ can be numerically determined by exhaustive search with 0,001 increment until the condition (1) is met.

\subsection{Continuous girder with unilateral constraints}

For this structure (Fig.1, c) the equations can be written as:

$$
M_{o p t}=\frac{-\left(l-2 z_{1}\right)^{3}-z_{1}^{3}+\left(l^{3}-2 l z_{1}^{2}+z_{1}^{3}\right) k}{4\left(3 l-4 z_{1}\right)} q \text {, }
$$

$$
\begin{gathered}
M_{1 \max }=\left[\frac{x k\left(l^{3}-2 l z_{1}^{2}-z_{1}^{3}\right)}{4 z_{1}\left(3 l-4 z_{1}\right)}\right] q+ \\
+\left[\frac{x\left(-l^{3}-6 l z_{1}^{2}+6 l^{2} z_{1}-z_{1}^{3}\right)}{4 z_{1}\left(3 l-4 z_{1}\right)}-\frac{x^{2}}{2}\right] q,
\end{gathered}
$$

at

$$
x=\frac{\left(l^{3}-2 l z_{1}^{2}+z_{1}^{3}\right) k-l^{3}-6 l z_{1}^{2}+6 l^{2} z_{1}-z_{1}^{3}}{4 z_{1}\left(3 l-4 z_{1}\right)},
$$

a)

b)

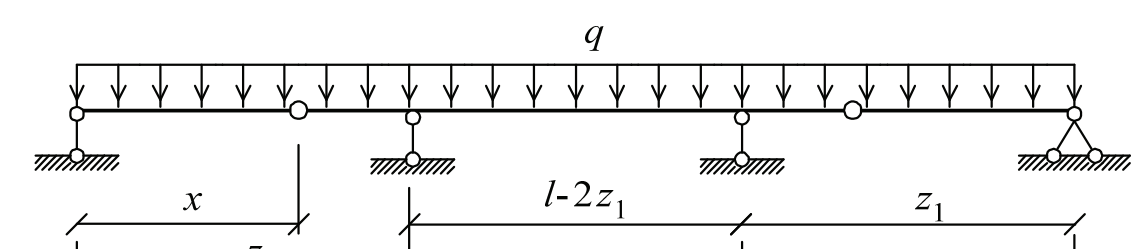

c)

d)

e)

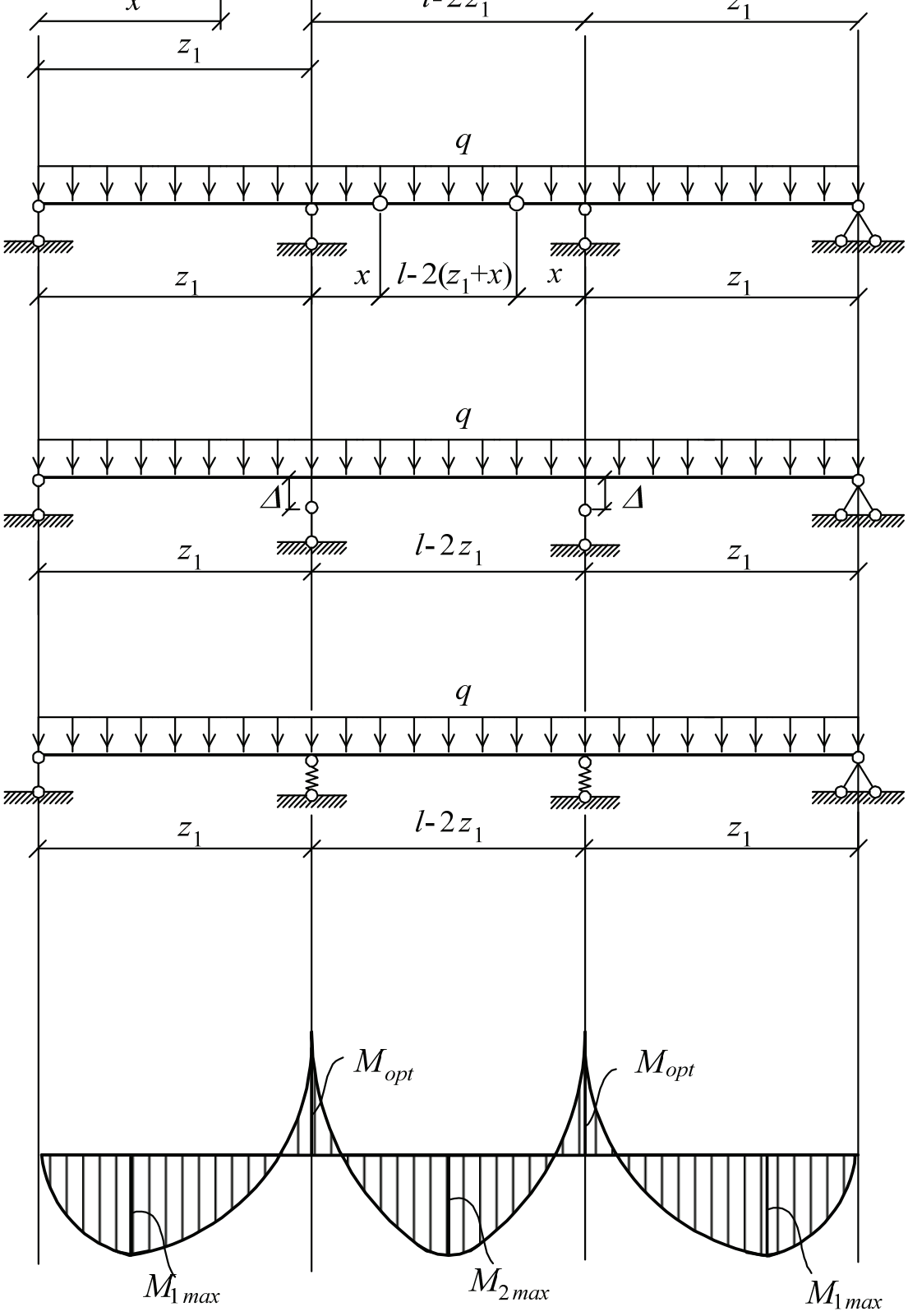

Fig. 1. Design options of the optimum structure and the optimum force distribution. 
Table 1. The results of optimization of the bridge.

\begin{tabular}{|c|c|c|c|c|c|c|c|}
\hline \multicolumn{2}{|c|}{ System type } & $z 1, \mathrm{~m}$ & $x, \mathrm{~m}$ & $\begin{array}{c}k \\
\text { or } c E J, \\
\mathrm{~m}^{3}\end{array}$ & $\begin{array}{c}M_{1 \text { max }}, \\
\mathrm{kNm}\end{array}$ & $\begin{array}{c}M_{\text {opt }} \mid, \\
\mathrm{kNm}\end{array}$ & $\begin{array}{c}M_{2 \text { max }}, \\
\mathrm{kNm}\end{array}$ \\
\hline $\begin{array}{c}\text { Statically determinate } \\
\text { three-span girders }\end{array}$ & hinges in the end spans & 11,351 & 9,404 & & 11,054 & 11,050 & 11,054 \\
\cline { 2 - 8 } & hinges in the middle span & 11,352 & 1,947 & & 11,058 & 11,049 & 11,049 \\
\hline \multirow{2}{*}{ Continuous girders } & $\begin{array}{c}\text { with unilateral support } \\
\text { constraints }\end{array}$ & 11,369 & & $\begin{array}{c}k \\
0,0271\end{array}$ & 10,994 & 10,996 & 10,989 \\
\cline { 2 - 8 } & with resilient constraints & 11,351 & & $\begin{array}{c}c E J \\
40,151\end{array}$ & 11,053 & 11,052 & 11,052 \\
\hline
\end{tabular}

$M_{2 \max }=\left[\frac{2\left(l^{3}-2 l z_{1}^{2}+z_{1}^{3}\right) k+l^{3}-4 l^{2} z_{1}+4 l z_{1}^{2}-2 z_{1}^{3}}{8\left(3 l-4 z_{1}\right)}\right] q$,

where coefficient $k$ determines part of the distributed load $k q$, at which the girder adjoins the supports, i.e. becomes statically determinate.

Parameters $z_{1}$ and $k$ can be numerically determined by exhaustive search with 0,001 and 0,0001 increment, respectively, until the conditions (1), (2) are met.

\subsection{Continuous girder on resilient supports}

Let us write the equations for the structure shown in Fig. 1, d:

$$
\begin{gathered}
M_{1 \max }=\frac{x X_{1}}{z_{1}}+\frac{q x\left(z_{1}-x\right)}{2} \text { at } x=\left(\frac{X_{1}}{z_{1}}+\frac{q z_{1}}{2}\right) / q, \\
M_{o p t}=X_{1} ; M_{2 \max }=X_{1}+\frac{q\left(l-2 z_{1}\right)^{2}}{8},
\end{gathered}
$$

where

$$
X_{1}=-\frac{\left(l-2 z_{1}\right)^{3}+z_{1}^{3}-12 c E J\left(l-z_{1}\right) / z_{1}}{3 l-4 z_{1}+6 c E J / z_{1}^{2} /\left(l-2 z_{1}\right)^{2}} \frac{q}{4} .
$$

Parameters $z_{1}, c E J$ can be numerically determined by exhaustive search with 0,001 and 0,0001 , respectively, until the conditions (1), (2) are met.

The results of optimization of all types of girders are summarized in Table 1. The calculations confirm the assertion that under constant load the optimal force distributions are practically equal for all four structures' types (Fig. 1, e).

\section{Conclusions}

As a result, the authors have proved that there are optimal solutions in the set of statically indeterminate systems both with unilateral and with elastic constraints, and under constant load they completely coincide with the solutions in the set of statically determinate systems.

\section{References}

1. A. Vinogradov, Problems of optimal design in structural mechanics (High school, 1973).

2. R. Bellman, Applied problem of dynamic programming (Science, 1965).

3. M. Reitman, Methods of optimal design of deformed bodies (Science, 1976)

4. N. Abovskiy, Proceeding international congress ICSS-98, 307-313 (1998).

5. N. Banichuk, Forms optimization of elastic solids (Science, 1980).

6. V. Gemintern, Methods of optimal design (Science, 1980)

7. M. Zhou, G. Rozvany, Comput Methods Appl Mech Eng, 89(1-3), 309-336, (1991).

8. M. Bendsoe, N. Kikuchi, Comput Methods Appl Mech Eng, 71(2), 197-224, (1988).

9. Y. Xie, G. Steven, Comput Struct, 49(5), 885-896, (1993).

10. K. Choi, N. Kim, Structural sensitivity analysis and optimization I-linear systems (Springer, 2005)

11. Z. Luo, N. Zhang, Y. Wang, W. Gao, Int J Numer Methods Eng 93(4), 443-464 (2013)

12. G. Vasilkov, Evolution theory of life cycle of mechanical systems (LKI, 2008).

13. Y. Kitov, G. Vatulia, M. Verevicheva, Collected scientific works of Ukrainian State University of Railway Transport, 143, 124 - 131 (2014).

14. Y. Kitov, G. Vatulia, Collected scientific works of Ukrainian State University of Railway Transport, 125, 24 - 33 (2011).

15. V. Shmukler, Collected scientific works of Ukrainian State University of Railway Transport, 167, $54-69$ (2017).

16. E. Chikhladze, G. Vatulia, Y. Kitov, Basis for calculation and design of composite and steelconcrete structures (Transport of Ukraine, 2006).

17. M. Gogol, Bridges and Tunnels: theory and practice, 3, 33-38 (2012). 